\title{
Stabilizing sulfur cathodes using nitrogen-doped graphene as a chemical immobilizer for Li-S batteries
}

Lu Li $^{1,2}$, Guangmin Zhou ${ }^{1}$, Lichang Yin ${ }^{1}$, Nikhil Koratkar ${ }^{2}$, Feng Li $^{1 *}$, and

\author{
Hui-Ming Cheng ${ }^{1}$
}

${ }^{1}$ Shenyang National Laboratory for Materials Science, Institute of Metal Research, Chinese Academy of Sciences, 72 Wenhua Road, Shenyang 110016, China.

${ }^{2}$ Department of Mechanical, Aerospace and Nuclear Engineering, Rensselaer Polytechnic Institute, $1108^{\text {th }}$ Street, Troy, NY 12180, USA.

\begin{abstract}
Lithium-sulfur (Li-S) battery, as a high energy-density storage system, has attracted great attention for next generation rechargeable battery applications. However, the fast capacity decay caused by lithium polysulfides (LiPSs) dissolution impedes its prospect for commercialization. In this work, nitrogen-doped graphene, as a chemical immobilizer, was designed to bind LiPSs and stabilize sulfur in the cathode for high performance Li-S batteries. The incorporated nitrogen dopants in the graphene network were found to have a strong binding effect on the LiPSs to improve electrochemical stability and promote fast electrochemical reaction kinetics. As a result, the nitrogen doped graphene-based sulfur electrode could deliver an initial capacity of $\sim 1200 \mathrm{mAh} \mathrm{g}^{-1}$ at $0.3 \mathrm{~A} \mathrm{~g}^{-1}$, and exhibit good capacity retention with only $0.05 \%$ capacity decay per cycle after 300 cycles at $0.75 \mathrm{~A} \mathrm{~g}^{-1}$.
\end{abstract}

${ }^{*}$ Corresponding author. Fax: +86 242390 3126. E-mail address: fli@imr.ac.cn (F. Li). 


\section{Introduction}

Lithium-sulfur ( $\mathrm{Li}-\mathrm{S})$ battery is a promising candidate for next generation energy storage systems due to its high theoretical specific capacity $\left(1675 \mathrm{mAh} \mathrm{g}^{-1}\right)$ and energy density $\left(\sim 2600 \mathrm{Wh} \mathrm{kg}^{-1}\right)$. Moreover, the low cost, environmental friendliness and wide availability of sulfur makes it attractive for battery applications [1-4]. However, the practical application of the $\mathrm{Li}-\mathrm{S}$ batteries has been impeded due to a series of obstacles such as low electrical conductivity of sulfur/lithium sulfides, low Coulombic efficiency caused by the dissolving and shuttling of soluble lithium polysulfides (LiPSs), and large volume expansion/shrinkage of sulfur during lithiation/delithiation processes [5-8].

To address the above issues, graphene based electrodes have attracted considerable interest for applications in $\mathrm{Li}-\mathrm{S}$ batteries due to their high electrical conductivity and large surface area [9], such as graphene wrapped sulfur cathode [10], three-dimensional hierarchical graphene network encapsulated sulfur electrode [11], unstacked double-layer graphene confined sulfur cathode [12], and graphene sandwiched sulfur structure [13]. As a high conductivity, porous and flexible framework, graphene was incorporated into the electrodes to overcome the shortcomings of $\mathrm{Li}-\mathrm{S}$ batteries, such as the insulating nature of sulfur, "shuttle effect" of LiPSs during repeated electrochemical charge/discharge cycles, and large volume expansion problems. However, these designs are still limited by the weak interactions (related to physical adsorption/confinement) between the graphene matrix and the sulfur species, which are not sufficient for alleviating polysulfides dissolution into the 
electrolyte. Besides hetero-atoms doping in the carbon materials as the strategy to bring chemical binding with lithium polysulfides, other promising chemical adsorbents such as transition metal oxides or sulphides have recently been introduced into Li-S battery system to immobilize lithium polysulfides [14-17]. However, the use of non-conductive metal oxides as polysulfide immobilizers will affect the rate performance of the electrodes. Therefore, an ideal conductive substrate should not only host non-polar sulfur, but also strongly adsorb (or bind) polar polysulfide species to extend the battery cycle life and achieve high utilization of active sulfur.

Generally, chemically-derived graphene from graphene oxide (GO) is decorated with abundant oxygen-containing functional groups (e.g. hydroxyl $(\mathrm{C}-\mathrm{OH})$, carboxyl $(-\mathrm{COOH})$, carbonyl $(-\mathrm{C}=\mathrm{O})$, epoxyl $(-\mathrm{C}-\mathrm{O}-\mathrm{C}-))$, and lattice defects (e.g. atom vacancy, distortion, and dangling bonds) on the lateral surface or at edges [18, 19]. It is well demonstrated that non-metal heteroatoms can be easily incorporated into the graphene network, which has proven to be a very effective approach to tune the electrical and chemical environment of the carbon surface for electrochemical reactions [20]. Among different non-metal heteroatoms, nitrogen $(\mathrm{N})$ is a very popular doping element that could act as a Lewis base in the lattice of graphene and interacts with other molecules [21], such as LiPSs and final discharge lithium sulfides, to significantly improve the electrochemical performance of $\mathrm{Li}-\mathrm{S}$ batteries. For example, $\mathrm{N}$-doped porous carbon [22, 23], $\mathrm{N}$-doped carbon nanotubes [24, 25] and $\mathrm{N}$-doped graphene [26-28] were applied as the conductive substrates to improve the performance of Li-S batteries. In these studies, Wang's group proposed that the 
existence of $\mathrm{N}$ in the mesoporous carbon will promote the formation of bonds between sulfur atoms and nitrogen-related functional groups on the carbon [22]. However, the effect of N-dopants on the soluble LiPSs that are generated during the electrochemical reaction of a Li-S battery are not well-understood. Further, it is still ambiguous as to which types of $\mathrm{N}$-dopants are the most effective immobilizers to restrict the dissolution of LiPSs and what is the detailed interaction mechanism between N-dopants and LiPSs.

Herein, we propose a facile strategy towards high-performance $\mathrm{Li}-\mathrm{S}$ battery by using $\mathrm{N}$-doped graphene as chemical immobilizer to stabilize sulfur and its discharge products. To provide the proof-of-the-concept, the raw material of GO was annealed in ammonia atmosphere serving as sulfur host (denoted as $\mathrm{N}-\mathrm{G}$ ), in side-by-side comparison to GO treated in argon (denoted as A-G). The results lead to insights about the roles played by the doped $\mathrm{N}$ heteroatom in graphene network on the electrochemical reaction. As a result, a high capacity of $\sim 1200 \mathrm{mAh}^{-1}$, excellent cycling stability, and good rate capability of a N-G-based Li-S battery are achieved.

\section{Experimental}

\subsection{Synthesis of GO}

GO was fabricated using natural graphite flakes by a modified Hummers' method [29]. The concentration of the GO suspension obtained was $1.8 \mathrm{mg} \mathrm{mL}^{-1}$.

\subsection{Synthesis of N-G and A-G}

$\mathrm{N}-\mathrm{G}$ was prepared by annealing $\mathrm{GO}$ at $400{ }^{\circ} \mathrm{C}$ under $\mathrm{NH}_{3}$ gas flow. Briefly, 100 mg of GO was put in the middle of a quartz tube furnace in $\mathrm{NH}_{3}$ flow of $50 \mathrm{sccm}$. It 
was then heated to $400{ }^{\circ} \mathrm{C}$ with a ramp of $5{ }^{\circ} \mathrm{C} \min ^{-1}$ and held at this temperature for $2 \mathrm{~h}$. The resultant N-G samples were obtained after the furnace was naturally cooled to room temperature. In order to prepare the A-G sample, GO was thermally reduced by a similar procedure under $400^{\circ} \mathrm{C}$ at argon atmosphere.

2.3 Synthesis of sulfur/graphene composites (N-G-S and A-G-S)

N-G-S and A-G-S were fabricated via a melt-diffusion method. The mass ratio of graphene based material (N-G or A-G) and sulfur is 3:7. The mixtures were co-heated at $155^{\circ} \mathrm{C}$ in a sealed flask for $4 \mathrm{~h}$ to obtain the N-G-S and A-G-S.

\subsection{Materials Characterisation}

Scanning electron microscopy (SEM) observations were performed on a FEI Nova NanoSEM 430 operated at $15 \mathrm{kV}$. Transmission electron microscopy (TEM) and STEM were performed using a Tecnai F20 (200 kV). Energy-dispersive X-ray spectroscopy (EDS) was used for elemental analysis. X-ray photoelectron spectroscopy (XPS) analysis was performed by using an ESCALAB 250 instrument with $\mathrm{Al} \mathrm{K} \alpha$ radiation $(15 \mathrm{kV}, 150 \mathrm{~W})$ under a pressure of $4 \times 10^{-8} \mathrm{~Pa}$. X-ray diffraction (XRD) was conducted with a D-MAX/2400 diffractometer $(\mathrm{Cu} \mathrm{K} \alpha, \lambda=0.154056 \mathrm{~nm})$. Raman spectra were collected with a $632.8 \mathrm{~nm}$ He-Ne laser with a Jobin Yvon LabRam HR800 micro-Raman system at room temperature. Thermogravimetric analysis (TGA) was performed with a NETZSCH STA 449C thermo-balance in argon with a heating rate of $10{ }^{\circ} \mathrm{C} \min ^{-1}$ to $500{ }^{\circ} \mathrm{C}$. Zeta potential was measured by the ZETASIZER Nano series (Nano-ZS90). $10 \mathrm{mg} \mathrm{N}-\mathrm{G}$ and A-G powders were dispersed in $50 \mathrm{~mL}$ distilled water and sonicated by a tip sonicator (SCIENTZ-IID) for $0.5 \mathrm{~h}$. The obtained graphene dispersion was then used to measure the Zeta potential. Fourier transform infrared spectroscopy (FTIR) spectra were obtained using a Bruker 
TENSOR 27 spectrometer. The samples were collected using the KBr-pellet method and the obtained spectra were normalized using OPUS 6.5 software.

\subsection{Electrochemical Measurements}

The N-G-S and A-G-S electrodes were prepared by mixing 80 wt $\%$ active material with 10 wt \% conductive carbon black (super P) as a conducting agent and 10 wt \% polyvinylidene fluoride dissolved in N-methyl-2-pyrrolidone as a binder to form a slurry, which was then coated onto a $\mathrm{Al}$ foil and dried under vacuum at $60^{\circ} \mathrm{C}$ for $12 \mathrm{~h}$. The obtained sulfur electrodes were shaped into a circular pellet with a diameter of $12 \mathrm{~mm}$ and was used as a cathode. The mass loading of the sulphur cathode was $\sim 2 \mathrm{mg} \quad \mathrm{cm}^{-2}$. The electrolyte was $1.0 \mathrm{M}$ lithium bis-trifluoromethanesulphonylimide in DOL and DME (1:1 by volume) with $0.5 \mathrm{wt} \%$ $\mathrm{LiNO}_{3}$ additive. 2032-type stainless steel coin cells were used to assemble test batteries. Lithium metal foil was used as the anode, and Celgard 2400 (thickness: 25 mm, porosity: $41 \%$ ) was used as the separator. The coin-type cell was assembled in an Ar-filled glove box (MBraun Unilab). A LAND galvanostatic charge-discharge instrument was used to perform the measurements. The current density set for tests was referred to the mass of sulfur in the cathode and was varied from 0.3 to $6 \mathrm{~A} \mathrm{~g} \mathrm{~g}^{-1}$. The charge/discharge voltage range was $1.5-2.8 \mathrm{~V}$. The cyclic voltammetry (CV) was measured using a VSP-300 multichannel potentiostat/galvanostat (Bio-Logic, France) workstation operated in the voltage range of $1.5-2.8 \mathrm{~V}\left(v s . \mathrm{Li}^{+} / \mathrm{Li}\right)$ at a scan rate of 0.1 $\mathrm{mV} \mathrm{s}{ }^{-1}$. Electrochemical impedance spectroscopy (EIS) measurements were performed in the frequency range of $100 \mathrm{kHz}$ to $0.01 \mathrm{~Hz}$ with an AC voltage amplitude of $10 \mathrm{mV}$ at the open-circuit potential or discharge to $2.3 \mathrm{~V}$ after five cycles. 


\section{Results and discussion}

The graphene-sulfur composites were prepared by thermal-diffused sulfur into N-G and A-G samples at $155^{\circ} \mathrm{C}[30,31]$. SEM images reveal that both N-G-S and A-G-S have a curly morphology with a thin, wrinkled laminated structure (Figure 1a,c and Figure S1a). The elemental composition was analyzed by EDS that shows the presence of $\mathrm{C}, \mathrm{O}$, and $\mathrm{S}$ in the N-G-S and A-G-S composites (Figure 1b, Figure S2), while N element only appears in the N-G-S sample, indicating the successful incorporation of $\mathrm{N}$ atoms into the carbon framework. The elemental distribution results obtained by the EDS elemental mappings (Figure S1b-d) and STEM (Figure 1d-g) further confirm the existence and homogeneous distribution of $\mathrm{C}, \mathrm{O}, \mathrm{S}$ and $\mathrm{C}, \mathrm{O}$, $\mathrm{S}, \mathrm{N}$ atoms on the surface of A-G-S and N-G-S composites. The uniform dispersed sulfur without obvious agglomeration suggests the well mixed state of sulfur in the composites, which will be beneficial for improving sulfur utilization.

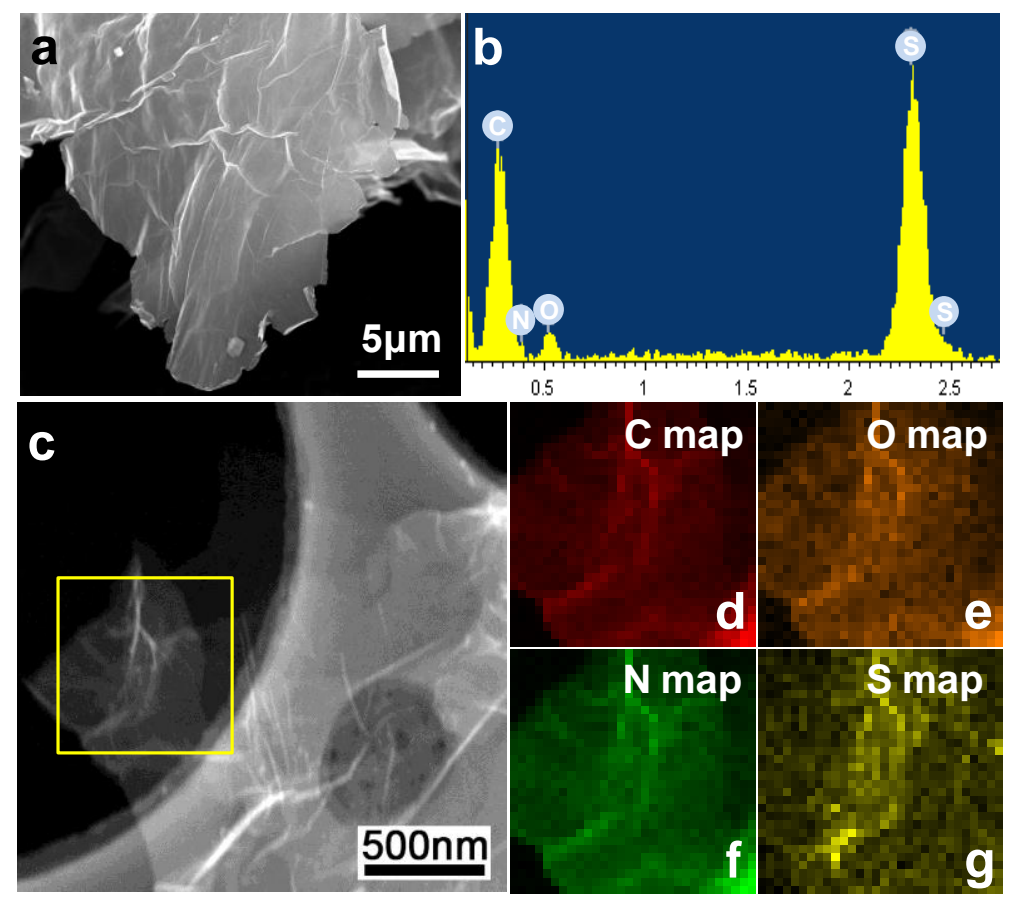

Figure 1. (a) SEM image of the N-G-S composite. (b) EDS spectrum of the N-G-S 
composite (unit: keV). (c) TEM image of the N-G-S composite. (d-g) Corresponding elemental mappings of the N-G-S composite in (c).

XRD patterns of the GO, A-G, N-G, A-G-S and N-G-S samples are shown in Figure S3. GO shows a typical diffraction peak at around $12^{\circ}$ [32]. After the heat treatment, this peak disappears while a broad diffraction peak emerges between 22 and $28^{\circ}$ in the XRD patterns for both $\mathrm{N}-\mathrm{G}$ and $\mathrm{A}-\mathrm{G}$ samples, suggesting the reduction reaction of GO. After impregnating sulfur through melt-diffusion strategy, all the pronounced diffraction peaks observed in the N-G-S and A-G-S composites, except for the broad one at around $26^{\circ}$, could be assigned to the orthorhombic sulfur (JCPDS card No. 08-0247) [33]. To verify the doping of nitrogen on the graphene surface, XPS was used to elucidate the surface chemical composition of the A-G, N-G, A-G-S and N-G-S samples. Figure 2a demonstrates the presence of $\mathrm{C} 1 \mathrm{~s}, \mathrm{~N} \mathrm{1s}$, and $\mathrm{O} 1 \mathrm{~s}$ peaks in the $\mathrm{N}-\mathrm{G}$ while only $\mathrm{C} 1 \mathrm{~s}$ and $\mathrm{O}$ 1s peaks existing in the A-G sample, indicating the incorporation of nitrogen into the $\mathrm{sp}^{2}$ carbon network of $\mathrm{N}-\mathrm{G}$. After impregnating sulfur, $S 2$ s and $S 2 p$ peaks could be detected in the A-G-S and N-G-S samples [34]. Based on the N1s peak that appeared at about $400 \mathrm{eV}$ after $\mathrm{NH}_{3}$ treatment, it is estimated that $\sim 7.1 \mathrm{wt} \%$ nitrogen was doped into the carbon framework, accompanied with a decrease in the oxygen content. The $\mathrm{C} / \mathrm{O}$ ratios are also investigated as shown in Figure 2b. The $\mathrm{C} / \mathrm{O}$ ratios are 2.5, 7.6 and 9.6 for GO, A-G, and N-G, respectively, which suggest a more efficient reduction process by using $\mathrm{NH}_{3}$ to treat $\mathrm{GO}$ than employing argon. The binding energy peaks observed in the high-resolution N 1s profile of the N-G-S at $\sim 398.3, \sim 399.8$ and $\sim 401.7 \mathrm{eV}$ (Figure 
2c) correspond to pyridinic, pyrrolic and graphitic nitrogen, respectively $[27,35]$. The C 1s spectra of the N-G-S sample consists of component peaks at $\sim 284.6 \mathrm{eV}$ and $\sim 285.9 \mathrm{eV}$, corresponding to the carbon species of C-C and C-N, respectively, which also confirms nitrogen doping into the carbon network (Figure 2d). Figure S4 presents the FTIR spectra of the GO, A-G-S and N-G-S samples. Consistent with the XPS analysis, the infrared vibration intensities of oxygen-containing bonds (C-O-C bonding at $1045 \mathrm{~cm}^{-1}, \mathrm{C}-\mathrm{O}$ bonding at $1215 \mathrm{~cm}^{-1}$ and $1394 \mathrm{~cm}^{-1}$, and $\mathrm{C}=\mathrm{O}$ bonding at $1725 \mathrm{~cm}^{-1}$ ) [36, 37] of A-G-S and N-G-S show a pronounced decrease due to the reduction of oxygen-containing functional groups heated in argon and ammonia atmosphere. The $\mathrm{C}-\mathrm{N}$ bonding $\left(1575 \mathrm{~cm}^{-1}\right)$ appears in the spectrum of the N-G-S sample [38], further indicating the successful incorporation of nitrogen into the carbon network. 

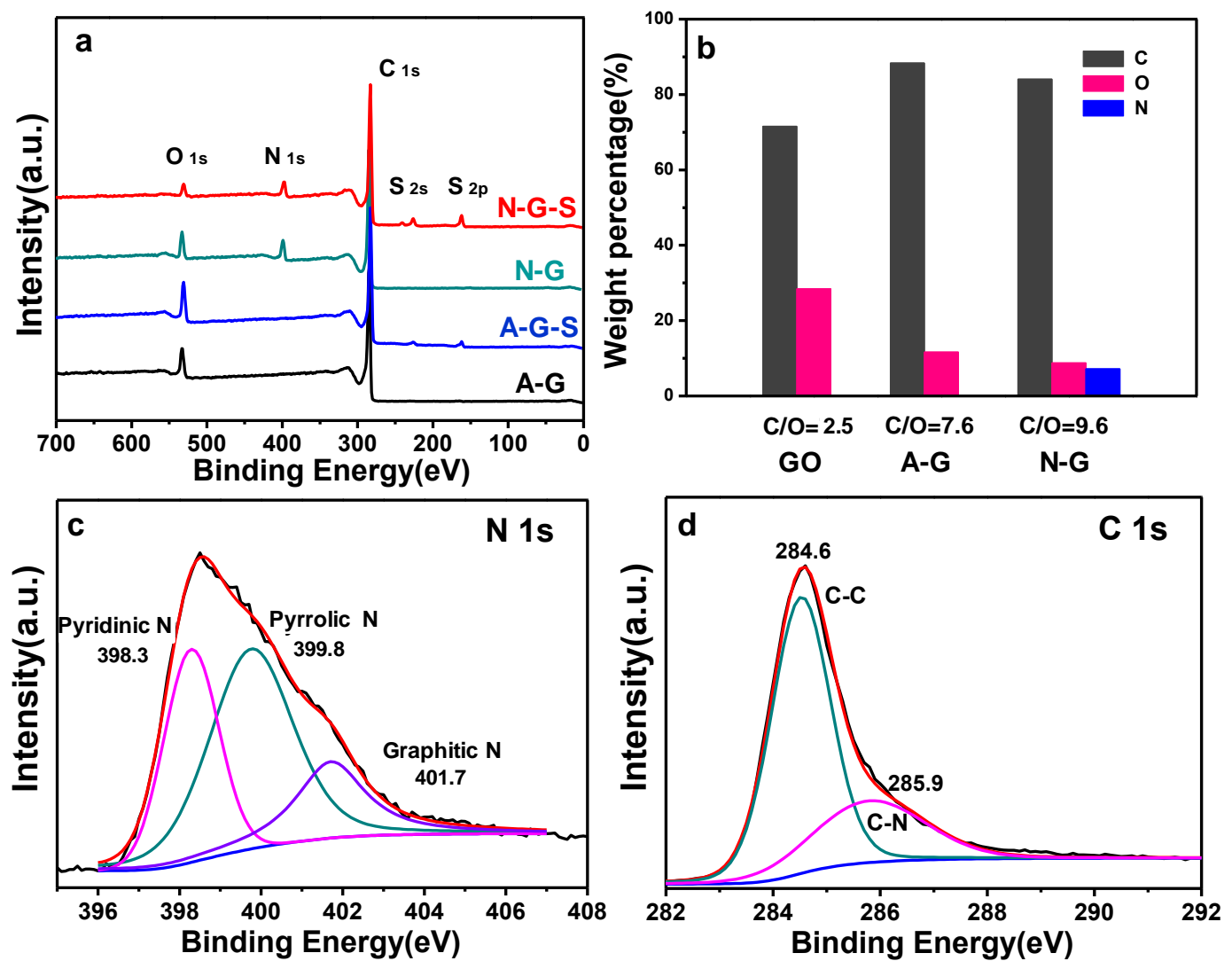

Figure 2. (a) XPS spectra of the surface chemical composition of the A-G, N-G, A-G-S and N-G-S samples. (b) Weight percentage of the $\mathrm{C}, \mathrm{O}, \mathrm{N}$, and $\mathrm{C} / \mathrm{O}$ ratios for the GO, A-G and N-G. (c) N 1s XPS spectrum of the N-G-S. (d) C 1s XPS spectrum of the N-G-S.

Raman spectroscopy is highly sensitive to the subtle structural variations in the carbon network and can be used to study the structural differences of the composite before and after reduction ( $\mathrm{GO}, \mathrm{A}-\mathrm{G}$ and $\mathrm{N}-\mathrm{G})$, and after mixing sulfur in the composite (A-G-S and N-G-S). All the samples display two distinct Raman peaks at $\sim 1335$ and $\sim 1580 \mathrm{~cm}^{-1}$, which correspond to the $\mathrm{D}$ band and $\mathrm{G}$ band of $\mathrm{sp}^{2}$ carbon materials, respectively [32]. As shown in Figure 3a, the intensity ratios of D band to G band $\left(\mathrm{I}_{D} / \mathrm{I}_{G}\right)$ of the $\mathrm{A}-\mathrm{G}\left(\mathrm{I}_{D} / \mathrm{I}_{G}=1.12\right)$ and $\mathrm{N}-\mathrm{G}\left(\mathrm{I}_{D} / \mathrm{I}_{G}=1.17\right)$ are higher than that of 
$\mathrm{GO}\left(\mathrm{I}_{D} / \mathrm{I}_{G}=1.07\right)$, which is possibly due to the increase of the number of $\mathrm{C}$ atoms at graphene edges or defects during the reduction process [39]. Owing to the nitrogen atoms doped in the network of the graphene, the ratio of $\mathrm{I}_{D} / \mathrm{I}_{G}$ of N-G is higher than that of A-G. Moreover, we have analyzed G peaks of the A-G, N-G, A-G-S and N-G-S samples in Figure 3a, the red shift (shift to higher frequency) of G peaks in the N-G and N-G-S samples compared to the un-doped A-G and A-G-S samples, further confirming the successfully N-doping in the samples [40]. After adding sulfur in the A-G and N-G, more defects lead to a higher intensity ratio between D band and G band in the A-G-S and N-G-S composites. The absence of the sulfur peaks (in the range of $100-500 \mathrm{~cm}^{-1}$ ) which stem from $\mathrm{S}-\mathrm{S}$ bond vibration in sulfur crystals, suggest that sulfur is well-dispersed in the A-G-S and N-G-S composites without long-range ordering [41]. TGA shows that the A-G-S and N-G-S composites (Figure 3b) have similar sulfur content $(\sim 67.1 \%$ and $\sim 68.6 \%$, respectively). However, compared with the A-G-S composite, the sulfur of N-G-S composite evaporates at an elevated temperature $\left(223^{\circ} \mathrm{C}\right.$ compared to $\sim 192{ }^{\circ} \mathrm{C}$ of A-G-S $)$, which is likely due to the strong interaction between nitrogen-doped graphene and sulfur [33]. Besides, we measured the Zeta potential of $\mathrm{A}-\mathrm{G}$ and $\mathrm{N}-\mathrm{G}$, to be $+0.254 \mathrm{mV}$ and $-1.951 \mathrm{mV}$, respectively, which suggest that $\mathrm{N}-\mathrm{G}$ should exhibit a better adsorption ability to $\mathrm{Li}_{2} \mathrm{~S}_{\mathrm{x}}$ $(4 \leq \mathrm{x} \leq 8)[27]$ 

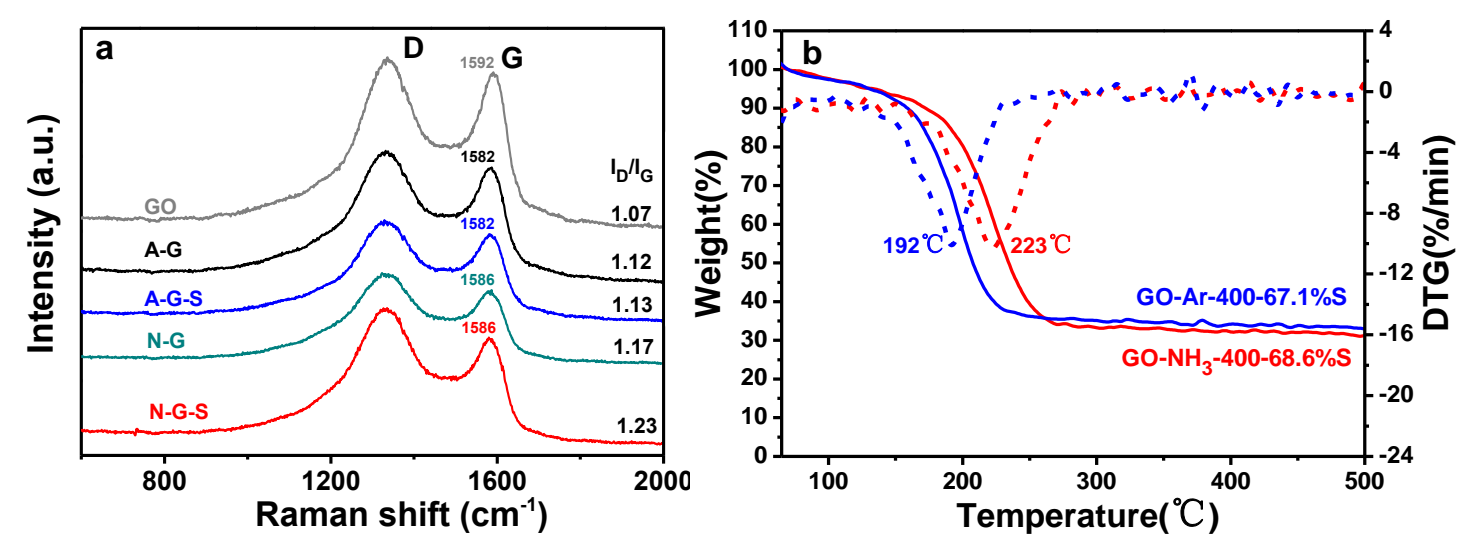

Figure 3. (a) Raman spectra of the GO, A-G, A-G-S, N-G and N-G-S samples. (b)

TGA and differential thermogravimetry (DTG) curves of similar sulfur loading in the A-G-S and N-G-S composites.

In order to prove the effect of the doped nitrogen atoms on the electrochemical performance of Li-S batteries, the A-G-S and N-G-S electrodes were tested in coin cells with a lithium-metal anode. Firstly, electrochemical impedance spectroscopy (EIS) was used to test the reaction kinetics of these cathodes. The Nyquist plots of these two battery systems at the open-circuit voltage are composed of a depressed semicircle in the high-medium frequency range, corresponding to the charge-transfer resistance $\left(\mathrm{R}_{\mathrm{ct}}\right)$ and a sloped tail in the low-frequency region, corresponding to the Warburg impedance related to the ion-diffusion process (Figure 4) [13, 42]. The $\mathrm{R}_{\mathrm{ct}}$ of the N-G-S electrode $(295.7 \Omega)$ is much lower compared to A-G-S $(939.4 \Omega)$ electrode, which can be attributed to the favorable charge- and ion-transfer processes from the graphene network and $\mathrm{N}$ dopants (Figure 4a). After five cycles and discharge to $2.3 \mathrm{~V}$, the curve for N-G-S consists two semicircles, the first semicircle (at high frequency) can be ascribed to the solid electrolyte interface (SEI) film resistance during cycling 
process and the second semicircle is due to the $\mathrm{R}_{\mathrm{ct}}$.[43] The SEI film resistance and $\mathrm{R}_{\mathrm{ct}}$ of N-G-S electrode are much smaller compared with A-G-S electrode due to the improved electronic transport process by $\mathrm{N}$-doping, the electrochemical conditioning effects such as better contacts and distribution of the polysulfide species on the graphene sheets (Figure 4b).
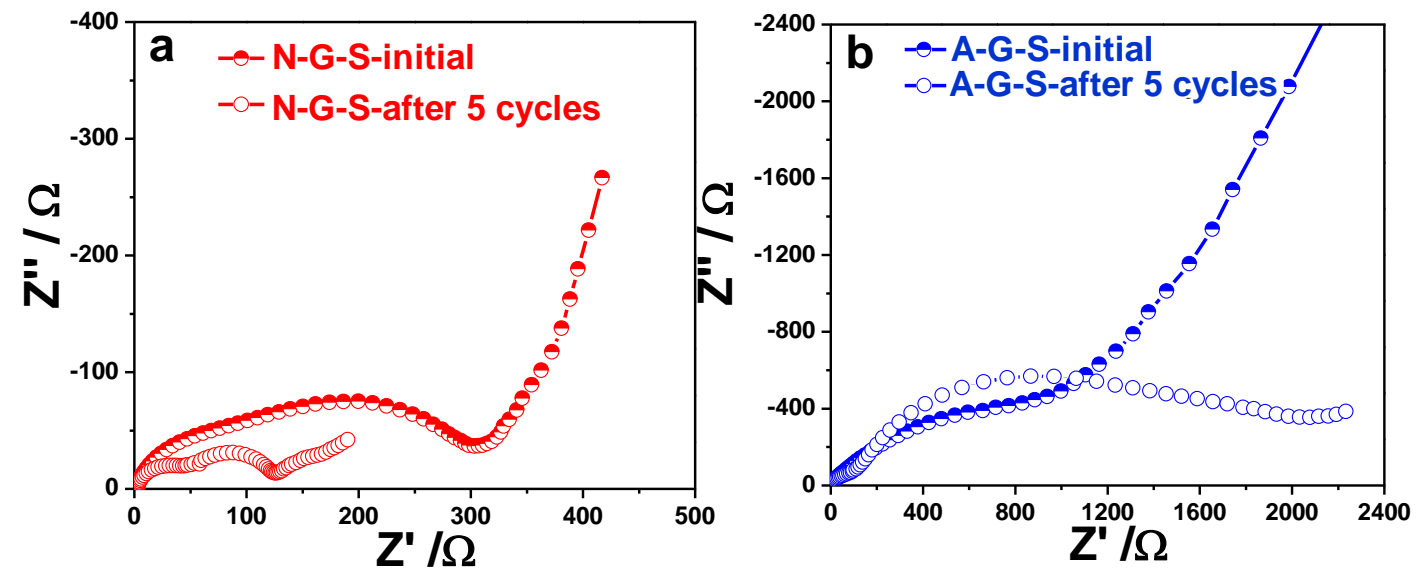

Figure 4. Nyquist plots of the (a) N-G-S and (b) A-G-S electrodes at the open-circuit potential and discharge to $2.3 \mathrm{~V}$ after five cycles with a frequency range of $100 \mathrm{kHz}$ to $0.01 \mathrm{~Hz}$ by using an AC voltage amplitude of $10 \mathrm{mV}$.

Figure 5a and $\mathrm{b}$ show the CV curves of the N-G-S and A-G-S electrodes scanned at a rate of $0.1 \mathrm{mV} \mathrm{s}^{-1}$. Two well-defined reduction peaks for $\mathrm{N}-\mathrm{G}-\mathrm{S}$ electrode exist distinctly in the first scan, which are located at 2.27 and $2.02 \mathrm{~V}$, corresponding to the two step conversions of sulfur to high-order LiPSs $\left(\operatorname{Li}_{2} \mathrm{~S}_{\mathrm{x}}, 4 \leq \mathrm{x} \leq 8\right)$ and high-order LiPSs to $\mathrm{Li}_{2} \mathrm{~S}_{2} / \mathrm{Li}_{2} \mathrm{~S}$, respectively [44]. Only one broad oxidation peak located at around $2.46 \mathrm{~V}$ is observed, showing the transformation of the lithium sulfides to soluble LiPSs and to the final sulfur $[13,45]$. At the same time, the reduction and 
oxidation peaks of A-G-S electrode are much broader than those of N-G-S electrode, which suggest the slower reaction kinetics, implying the sluggish and incomplete utilization of active materials. By comparing Figure 5a and b, we come to the conclusion that nitrogen-doped graphene plays an important role in suppressing the diffusion of polysulfide species and minimizing the mass loss of active materials during cycling. From the second cycle in Figure 5a, the cathodic peaks shift to a higher potential of about 2.32 and $2.05 \mathrm{~V}$, while the oxidation peaks also shift to a lower potential of about $2.42 \mathrm{~V}$ and the $\mathrm{CV}$ curves exhibit similar shapes, indicating that the reacting kinetics have been improved for the subsequent cycles and good reversibility is achieved for the N-G-S electrode. To better understand the reaction dynamics, we also depicted the peak potential and onset potential of N-G-S electrode and A-G-S electrode, as shown in Figure S5. The peak potential obtained from the second cycle of $\mathrm{CV}$ curve indicates the increased redox kinetics and smaller polarization for the N-G-S electrode due to the higher reduction peaks and lower oxidation peak. The onset potential also reveals that the existence of heteroatom will help to accelerate the oxidation reaction. Besides, the intensity of redox peaks from the A-G-S electrode drops constantly, indicating the occurrence of irreversible reactions (Figure 5b).

The rate capability of the N-G-S and A-G-S electrodes was investigated as shown in Figure 5c. The results show that N-G-S electrode exhibits a capacity above $1150 \mathrm{~mA} \mathrm{~h} \mathrm{~g}^{-1}$, much higher than that of the A-G-S electrode $\left(839 \mathrm{~mA} \mathrm{~h} \mathrm{~g}^{-1}\right)$ in the first five cycles at $0.3 \mathrm{~A} \mathrm{~g}^{-1}$. Upon increasing the current density, the capacities of the 
N-G-S electrode are much better than A-G-S electrode, and the specific capacity of the N-G-S electrode still remains at $\sim 330 \mathrm{~mA} \mathrm{~h}^{-1}$ under a very high current density $\left(6 \mathrm{~A} \mathrm{~g}^{-1}\right)$. We have also depicted the galvanostatic charge/discharge curves for the N-G-S and A-G-S electrodes, respectively, at different current densities. As shown in the Figure S6, we can see the difference that the polarization between charge and discharge curves of the A-G-S electrode is obviously larger than that of the N-G-S electrode under the same current density. These results indicate the introduction of heteroatoms not only creates much active sites for trapping lithium polysulfides, but also significantly reduces the charge transfer resistance. The cyclic stability of the samples was also investigated at a current density of $0.3 \mathrm{~A} \mathrm{~g}^{-1}$, as shown in Figure $\mathbf{5 d}$. The initial capacity of both electrodes is similar, however, A-G-S electrode showed a significant capacity decrease after only the initial few cycles. After 100 cycles, the N-G-S electrode exhibits good cyclic performance and retained a capacity of $\sim 880$ $\mathrm{mA} \mathrm{h}^{-1}$. Figure 5e shows the long cycling performance and Coulombic efficiency of A-G-S and N-G-S electrodes at a current density of $0.75 \mathrm{~A} \mathrm{~g}^{-1}$. After 300 cycles, the Coulombic efficiency of N-G-S electrode is still around $95 \%$ and the specific capacity is maintained above $700 \mathrm{~mA} \mathrm{~h} \mathrm{~g}^{-1}$ with only $\sim 0.051 \%$ capacity decay per cycle, much higher than A-G-S electrode with a Coulombic efficiency of only $85 \%$ and a capacity of $230 \mathrm{~mA} \mathrm{~h} \mathrm{~g}^{-1}(0.201 \%$ capacity decay per cycle $)$ under the same test condition. These results demonstrate that electrodes with nitrogen doping in the graphene network: (a) improve electron transport pathways with decreased resistance and increased reaction kinetics, and (b) provide strong binding between doped functional 
groups and $\mathrm{Li}_{2} \mathrm{~S} / \mathrm{LiPSs}$ to suppress polysulfide dissolution. The interactions between LiPSs and N-G with different doping configurations by density functional theory calculations were carried out in our group [46]. It was found that, among all different $\mathrm{N}$-doping configurations in $\mathrm{N}-\mathrm{G}$, only the clustered pyridinic $\mathrm{N}$-dopants can strongly attract LiPSs with large enough binding energies to effectively anchor the soluble LiPSs, due to (i) an enhanced attraction between Li ions in LiPSs and pyridinic $\mathrm{N}$-dopants and/or (ii) an additional attraction between $\mathrm{S}$ anions in LiPSs and Li ions captured by pyridinic N-dopants. 

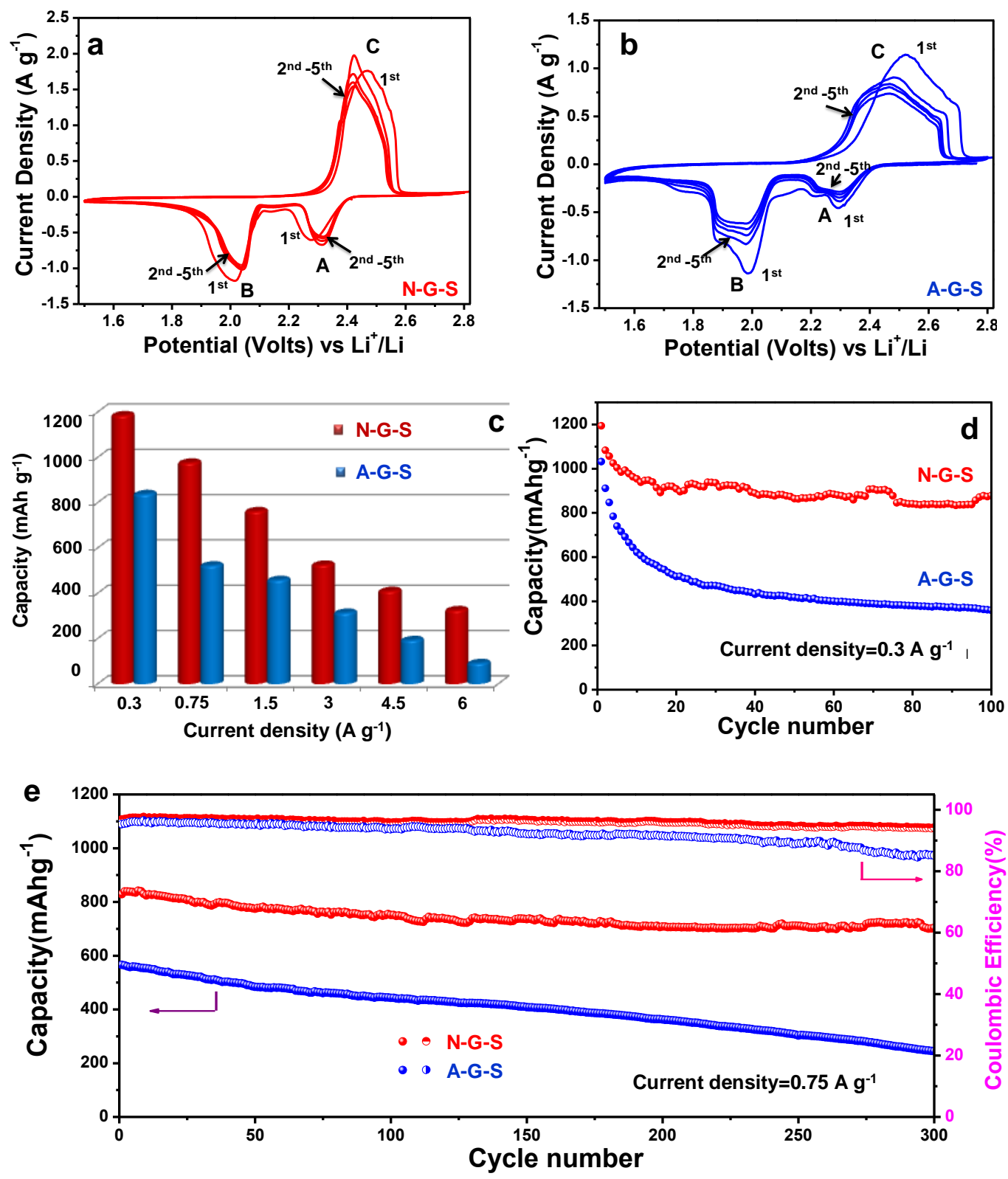

Figure 5. CVs of the (a) N-G-S electrode and (b) A-G-S electrode at a scan rate of 0.1 $\mathrm{mV} \mathrm{s}^{-1}$. (c) Rate performance, (d) cycling performance at a current density of $0.3 \mathrm{~A} \mathrm{~g}^{-1}$ and (e) long term cycling test at a current density of $0.75 \mathrm{~A} \mathrm{~g}^{-1}$ showing specific capacity and Coulombic efficiency of the Li-S batteries with N-G-S and A-G-S electrodes. 


\section{Conclusion}

In summary, a stabilized sulfur cathode was achieved by using nitrogen modified graphene as chemical immobilizer for Li-S batteries. The N-doped graphene cathode with $\sim 70 \%$ of sulfur exhibits a high specific capacity, fast reaction dynamics, good rate performance, and stable cycling performance with only $0.051 \%$ capacity decay per cycle up to 300 cycles. When the current density increased to $6 \mathrm{~A} \mathrm{~g}^{-1}$, the specific capacity is still retained up to $\sim 330 \mathrm{~mA} \mathrm{~h} \mathrm{~g}^{-1}$. The enhanced performance can be attributed to the facilitated electron and ion transportation, and the strong interactions between the doped graphene network and LiPSs, thereby reducing polysulfide dissolution. The related chemical adsorption insight obtained in this work could also pave the way in developing novel types of doped carbon frameworks as cathodes in Li-S batteries.

\section{Acknowledgements}

This work was supported by MOST (2014CB932402) and National Science Foundation of China (Nos.51521091, 51525206, 51172239, 51372253 and U1401243), "Strategic Priority Research Program" of the Chinese Academy of Sciences (XDA01020304, XDA09010104), the Key Research Program of the Chinese Academy of Sciences (Grant No. KGZD-EW-T06), and Research Supported by the CAS/SAFEA International Partnership Program for Creative Research Teams.

\section{References}

[1] Bruce PG, Freunberger SA, Hardwick LJ, Tarascon J-M. Li- $\mathrm{O}_{2}$ and Li-S batteries 
with high energy storage. Nat Mater. 2012;11(1):19-29.

[2] Yang Y, Zheng G, Cui Y. Nanostructured sulfur cathodes. Chem Soc Rev 2013;42(7):3018-32.

[3] Manthiram A, Fu Y, Chung S-H, Zu C, Su Y-S. Rechargeable Lithium-Sulfur Batteries. Chem Rev. 2014;114(23):11751-87.

[4] Goodenough JB. Energy storage materials: A perspective. Energy Storage Materials. 2015;1:158-61.

[5] Evers S, Nazar LF. New Approaches for High Energy Density Lithium-Sulfur Battery Cathodes. Acc Chem Res. 2013;46(5):1135-43.

[6] Wang D-W, Zeng Q, Zhou GM, Yin L, Li F, Cheng H-M, et al. Carbon-sulfur composites for Li-S batteries: status and prospects. $J$ Mater Chem A. 2013;1(33):9382-94.

[7] Liang J, Sun Z-H, Li F, Cheng H-M. Carbon materials for Li-S batteries: Functional evolution and performance improvement. Energy Storage Materials. 2016;2:76-106.

[8] Huang J-Q, Zhang Q, Wei F. Multi-functional separator/interlayer system for high-stable lithium-sulfur batteries: Progress and prospects. Energy Storage Materials. 2015;1:127-45.

[9] Yu M, Li R, Wu M, Shi G. Graphene materials for lithium-sulfur batteries. Energy Storage Materials. 2015;1:51-73.

[10]Wang HL, Yang Y, Liang YY, Robinson JT, Li YG, Jackson A, et al. Graphene-Wrapped Sulfur Particles as a Rechargeable Lithium-Sulfur Battery Cathode Material with High Capacity and Cycling Stability. Nano Lett. 2011;11(7):2644-7.

[11]Lv W, Li Z, Zhou G, Shao J-J, Kong D, Zheng X, et al. Tailoring Microstructure of Graphene-Based Membrane by Controlled Removal of Trapped Water Inspired by the Phase Diagram. Adv Funct Mater. 2014;24(22):3456-63.

[12]Zhao M-Q, Zhang Q, Huang J-Q, Tian G-L, Nie J-Q, Peng H-J, et al. Unstacked double-layer templated graphene for high-rate lithium-sulphur batteries. Nat Commun. 2014;5:3410.

[13]Zhou GM, Pei S, Li L, Wang D-W, Wang S, Huang K, et al. A Graphene-Pure-Sulfur Sandwich Structure for Ultrafast, Long-Life Lithium-Sulfur Batteries. Adv Mater. 2014;26(4):625-31.

[14] Yuan Z, Peng H-J, Hou T-Z, Huang J-Q, Chen C-M, Wang D-W, et al. Powering Lithium-Sulfur Battery Performance by Propelling Polysulfide Redox at Sulfiphilic Hosts. Nano Lett. 2016;16(1):519-27.

[15] Tao X, Wang J, Liu C, Wang H, Yao H, Zheng G, et al. Balancing surface adsorption and diffusion of lithium-polysulfides on nonconductive oxides for lithium-sulfur battery design. Nat Commun. 2016;7:11203.

[16]Zhou GM, Zhao Y, Zu C, Manthiram A. Free-standing $\mathrm{TiO}_{2}$ nanowire-embedded graphene hybrid membrane for advanced Li/dissolved polysulfide batteries. Nano Energy. 2015;12(0):240-9.

[17]Gao J, Li L, Tan J, Sun H, Li B, Idrobo JC, et al. Vertically Oriented Arrays of ReS2 Nanosheets for Electrochemical Energy Storage and Electrocatalysis. Nano Lett. 
2016;16(6):3780-7.

[18]Chen C-M, Zhang Q, Zhao X-C, Zhang B, Kong Q-Q, Yang M-G, et al. Hierarchically aminated graphene honeycombs for electrochemical capacitive energy storage. J Mater Chem. 2012;22(28):14076-84.

[19]Zhu YW, Murali S, Cai WW, Li XS, Suk JW, Potts JR, et al. Graphene and Graphene Oxide: Synthesis, Properties, and Applications. Adv Mater. 2010;22(35):3906-24.

[20] Su DS, Zhang J, Frank B, Thomas A, Wang X, Paraknowitsch J, et al. Metal-Free Heterogeneous Catalysis for Sustainable Chemistry. ChemSusChem. 2010;3(2):169-80.

[21]Ting-Zheng H, Hong-Jie P, Jia-Qi H, Qiang Z, Bo L. The formation of strong-couple interactions between nitrogen-doped graphene and sulfur/lithium (poly)sulfides in lithium-sulfur batteries. 2D Materials. 2015;2(1):014011.

[22] Song J, Xu T, Gordin ML, Zhu P, Lv D, Jiang Y-B, et al. Nitrogen-Doped Mesoporous Carbon Promoted Chemical Adsorption of Sulfur and Fabrication of High-Areal-Capacity Sulfur Cathode with Exceptional Cycling Stability for Lithium-Sulfur Batteries. Adv Funct Mater. 2014;24(9):1243-50.

[23] Sun F, Wang J, Chen H, Li W, Qiao W, Long D, et al. High Efficiency Immobilization of Sulfur on Nitrogen-Enriched Mesoporous Carbons for $\mathrm{Li}-\mathrm{S}$ Batteries. ACS Appl Mater Interfaces. 2013;5(12):5630-8.

[24]Peng H-J, Hou T-Z, Zhang Q, Huang J-Q, Cheng X-B, Guo M-Q, et al. Strongly Coupled Interfaces between a Heterogeneous Carbon Host and a Sulfur-Containing Guest for Highly Stable Lithium-Sulfur Batteries: Mechanistic Insight into Capacity Degradation. Adv Mater Interfaces. 2014;1(7):1400227.

[25] Wang Z, Niu X, Xiao J, Wang C, Liu J, Gao F. First principles prediction of nitrogen-doped carbon nanotubes as a high-performance cathode for Li-S batteries. RSC Adv. 2013;3(37):16775-80.

[26]Qiu Y, Li W, Zhao W, Li G, Hou Y, Liu M, et al. High-Rate, Ultralong Cycle-Life Lithium/Sulfur Batteries Enabled by Nitrogen-Doped Graphene. Nano Lett. 2014;14(8):4821-7.

[27]Zhou G, Paek E, Hwang GS, Manthiram A. Long-life Li/polysulphide batteries with high sulphur loading enabled by lightweight three-dimensional nitrogen/sulphur-codoped graphene sponge. Nat Commun. 2015;6:7760.

[28]Wang Z, Dong Y, Li H, Zhao Z, Bin Wu H, Hao C, et al. Enhancing lithium-sulphur battery performance by strongly binding the discharge products on amino-functionalized reduced graphene oxide. Nat Commun. 2014;5:5002.

[29]Zhao J, Pei S, Ren W, Gao L, Cheng H-M. Efficient Preparation of Large-Area Graphene Oxide Sheets for Transparent Conductive Films. ACS Nano. 2010;4(9):5245-52.

[30]Ji XL, Lee KT, Nazar LF. A highly ordered nanostructured carbon-sulphur cathode for lithium-sulphur batteries. Nat Mater. 2009;8(6):500-6.

[31]Wang D-W, Zhou GM, Li F, Wu K-H, Lu GM, Cheng H-M, et al. A microporous-mesoporous carbon with graphitic structure for a high-rate stable sulfur cathode in carbonate solvent-based Li-S batteries. Phys Chem Chem Phys. 
2012;14(24):8703-10.

[32]Pei S, Cheng H-M. The reduction of graphene oxide. Carbon. 2012;50(9):3210-28.

[33]Zhou G, Zhao Y, Manthiram A. Dual-Confined Flexible Sulfur Cathodes Encapsulated in Nitrogen-Doped Double-Shelled Hollow Carbon Spheres and Wrapped with Graphene for Li-S Batteries. Adv Energy Mater. 2015;5(9):1402263.

[34] Yang J, Xie J, Zhou X, Zou Y, Tang J, Wang S, et al. Functionalized N-Doped Porous Carbon Nanofiber Webs for a Lithium-Sulfur Battery with High Capacity and Rate Performance. J Phys Chem C. 2014;118(4):1800-7.

[35]Wu Z-S, Winter A, Chen L, Sun Y, Turchanin A, Feng X, et al. Three-Dimensional Nitrogen and Boron Co-doped Graphene for High-Performance All-Solid-State Supercapacitors. Adv Mater. 2012;24(37):5130-5.

[36] Stankovich S, Piner RD, Nguyen ST, Ruoff RS. Synthesis and exfoliation of isocyanate-treated graphene oxide nanoplatelets. Carbon. 2006;44(15):3342-7.

[37]Zhou GM, Wang D-W, Yin L-C, Li N, Li F, Cheng H-M. Oxygen Bridges between NiO Nanosheets and Graphene for Improvement of Lithium Storage. ACS Nano. 2012;6(4):3214-23.

[38]Xue Y, Liu J, Chen H, Wang R, Li D, Qu J, et al. Nitrogen-Doped Graphene Foams as Metal-Free Counter Electrodes in High-Performance Dye-Sensitized Solar Cells. Angew Chem Int Ed. 2012;51(48):12124-7.

[39]Stankovich S, Dikin DA, Piner RD, Kohlhaas KA, Kleinhammes A, Jia Y, et al. Synthesis of graphene-based nanosheets via chemical reduction of exfoliated graphite oxide. Carbon. 2007;45(7):1558-65.

[40]Imran Jafri R, Rajalakshmi N, Ramaprabhu S. Nitrogen doped graphene nanoplatelets as catalyst support for oxygen reduction reaction in proton exchange membrane fuel cell. J Mater Chem. 2010;20(34):7114-7.

[41]Dong Y, Liu S, Wang Z, Liu Y, Zhao Z, Qiu J. Sulfur-infiltrated graphene-backboned mesoporous carbon nanosheets with a conductive polymer coating for long-life lithium-sulfur batteries. Nanoscale. 2015;7(17):7569-73.

[42]Li L, Wu ZP, Sun H, Chen D, Gao J, Suresh S, et al. A Foldable Lithium-Sulfur Battery. ACS Nano. 2015;9(11):11342-50.

[43]Zhang SS, Xu K, Jow TR. The low temperature performance of Li-ion batteries. J Power Sources. 2003;115(1):137-40.

[44]Xiao LF, Cao YL, Xiao J, Schwenzer B, Engelhard MH, Saraf LV, et al. A Soft Approach to Encapsulate Sulfur: Polyaniline Nanotubes for Lithium-Sulfur Batteries with Long Cycle Life. Adv Mater. 2012;24(9):1176-81.

[45]Zhou GM, Yin L-C, Wang D-W, Li L, Pei S, Gentle IR, et al. Fibrous Hybrid of Graphene and Sulfur Nanocrystals for High-Performance Lithium-Sulfur Batteries. ACS Nano. 2013;7(6):5367-75.

[46] Yin L-C, Liang J, Zhou G-M, Li F, Saito R, Cheng H-M. Understanding the interactions between lithium polysulfides and $\mathrm{N}$-doped graphene using density functional theory calculations. Nano Energy. 2016;25:203-10. 


\section{Graphical Abstract:}

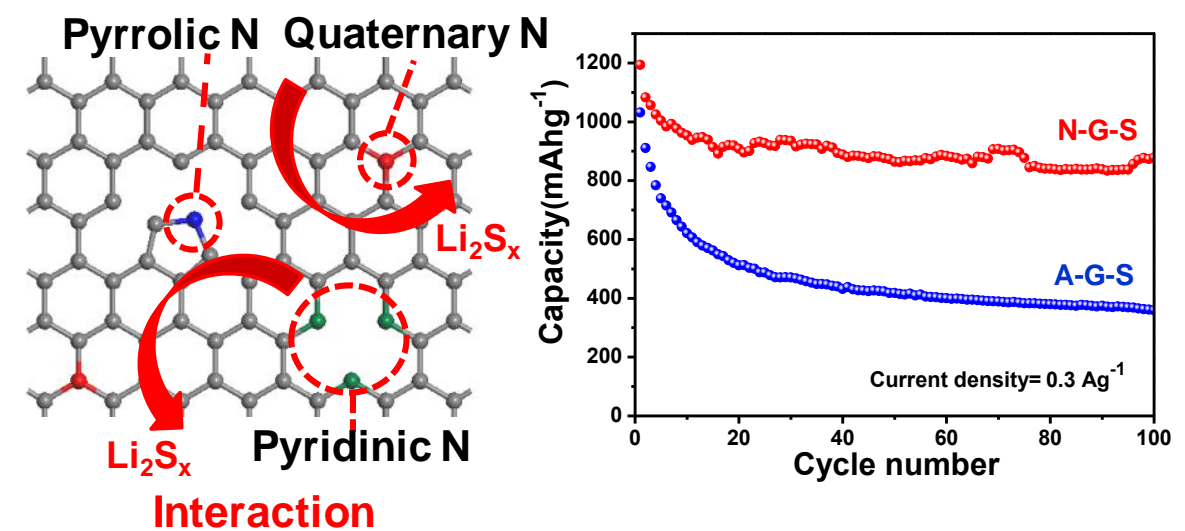

High performance Li-S batteries was designed by using nitrogen-doped graphene as a chemical immobilizer to bind lithium polysulfides and stabilize sulfur in the cathode. 